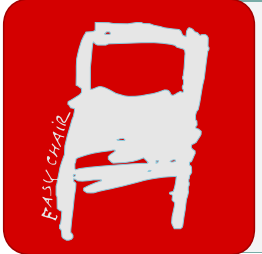

EPiC Series in Health Sciences

Volume 1, 2017, Pages 375-380

CAOS 2017. 17th Annual Meeting of the International

Society for Computer Assisted Orthopaedic Surgery

\title{
Patient-Specific Templates for Corrective Osteotomy
}

\author{
Ahmed Abdel Moghny and Mahmoud Hafez \\ The Orthopaedic Department, October 6 University, Giza, Egypt
}

\begin{abstract}
Correction osteotomy using conventional techniques may lead to under and over correction. The advantage of using patient specific templates for osteotomy is to predetermine the exact direction and degree of deformity before surgery and to estimate postoperative limb alignment which must mimic the physiologic loading to secure favorable clinical outcome.
\end{abstract}

Introduction

High tibial osteotomy using patient-specific templates (PST) aims at removing the isolated chondral defects at the articulating surfaces and to calculating the force and pressure before surgery [1]. Postoperative alignment and cartilage repair could be determined preoperatively. The use of PST could eliminate overload of the medial or lateral compartment as well as medial or lateral meniscus loss. It can also control cartilage damage in the medial or lateral femoro/ tibial condyles, and it can be used for initial osteoarthrosis in the medial or lateral compartments or at Tibia vara [2]. 
The aim of this work is to demonstrate the use of PST in high tibial osteotomy and corrective osteotomy for shaft deformity.

Methods:

PST was designed for high tibial osteotomy and also for corrective osteotomy of shaft deformity. The technique was applied in unilateral and bilateral high tibial osteotomy and tibial shaft deformity. The PST was based on CT-scan images which are transferred to specialized software to construct 3D models of bone. Virtual plan of surgery was done to design the templates which were used to perform the surgery. 3D printing machine is used to produce PST.

Two osteotomy lines were drawn: one to correct joint line and another line to correct genovarum. These joint lines as well as the angle of varum or valgum and the tibial slope were checked intraoperatively.

Results:

High tibial osteotomy for unilateral and bilateral was satisfactory in achieving the planned alignment with restoring normal function, correction of the deformity clinically and healing of the osteotomy. Corrective osteotomy for shaft deformity was achieved, and good alignment was obtained in coronal and sagittal planes. However the healing process in not completed after three months of surgery.

\section{Discussion:}

Skeletal deformities and arthritis are commonly associated with each other and sometimes it becomes confusing which is the cause or the result. In arthritic joints, the location of deformities could be around the joint or in the shafts of long bones [3]. There are different trends for treating arthritis that are associated with 
deformities particularly, those around the joint. In developing countries, there is a trend to treat osteoarthritis and correct deformities by osteotomies. In developed countries, the trend is to treat arthritis by joint replacement and corrective osteotomies according to the deformities' location. In addition, there are other factors that contribute to choosing the appropriate treatment option such as age, morbidity type of deformity [4]. At the end, accuracy and precision are required for preoperative planning and surgical implementation. PST used for osteotomy as a minimally invasive procedure. It allowed repositioning healthy bone and cartilage at the weight-bearing positions. In this work, osteotomy was done at the proximal tibia to provide optimal correction of the deformity, quick recovery and evaluation of the articulating bones preoperatively [5].

Conclusion:

PST can be used for correction of high tibial osteotomy and shaft deformities. It achieved good results of returning the normal function with high accuracy. The technique was guiding the surgeons to the correct cutting plane with optimum size of wedges and plates.

Disclosure

None

References

1. Paley D, Bhatnagar J, Herzenberg JE, Bhave A. New procedures for tightening knee collateral ligaments in conjunction with knee realignment osteotomy. Orthop Clin North Am 1994;25:533-55.

2. Fehring TK, Odum S, Griffin WL, Mason JB, Nadaud M. Early failure in total knee arthroplasty. Clin Orthop Relat Res 2001;392:315-8. 
3. Sharkey PF, Hozack WJ, Rothman RH, Shastri S, Jacoby SM. Insall Award paper. Why are knee replacements failing today? Clin Orthop Relat Res 2002;404:7-13.

4. Chillag KJ Nichollas PJ. High tibial osteotomy: A retrospective analysis of 30 cases. Orthopaedics 1984;7:1821.

5. Coventry MB, Ilstrup DM, Wallrichs SL. Proximal tibial osteotomy. A critical long-term study of eighty-seven cases. The Journal of Bone and Joint Surgery 1993;75-A-2:196-201.

Figures:

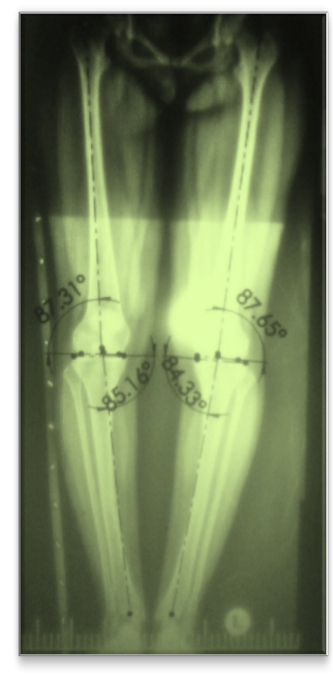


Fig (1): preoperative X-ray

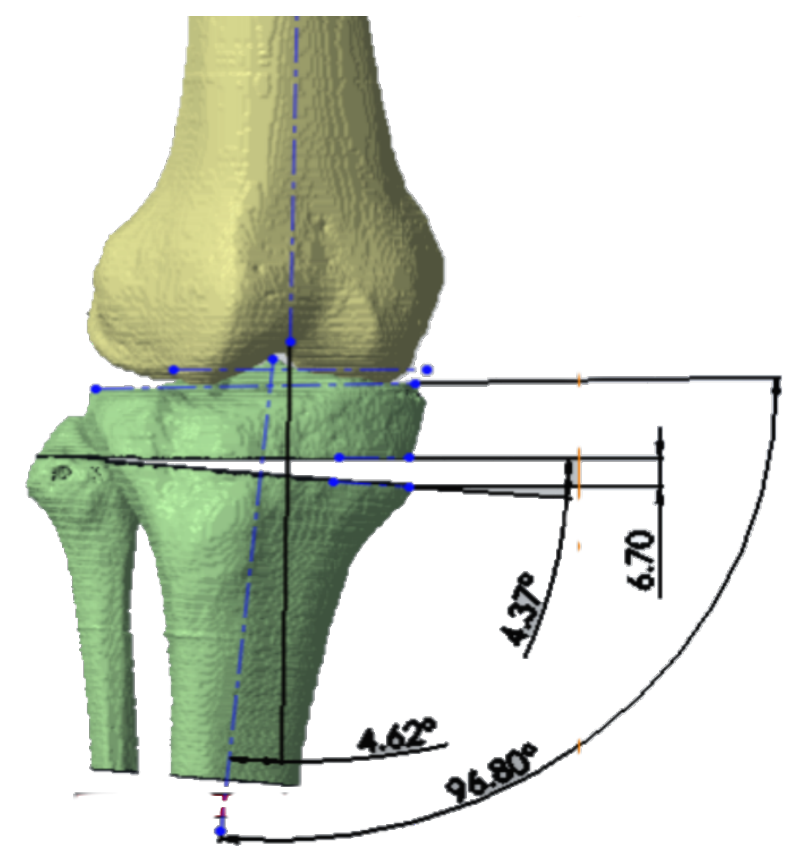

Fig (2): Bone reconstruction and planning
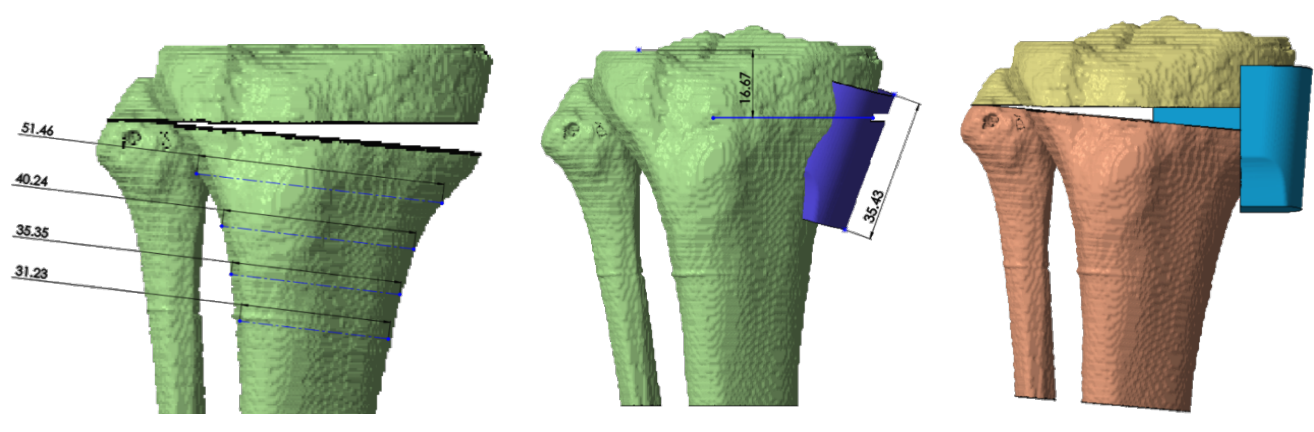
Fig (3): Surgery planning of high tibial osteotomy
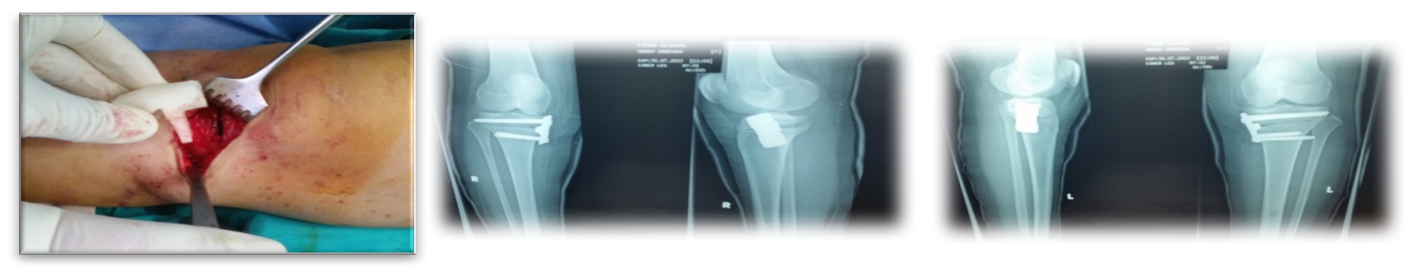

Fig (4): Intraoperative and postoperative x-ray (bilateral)
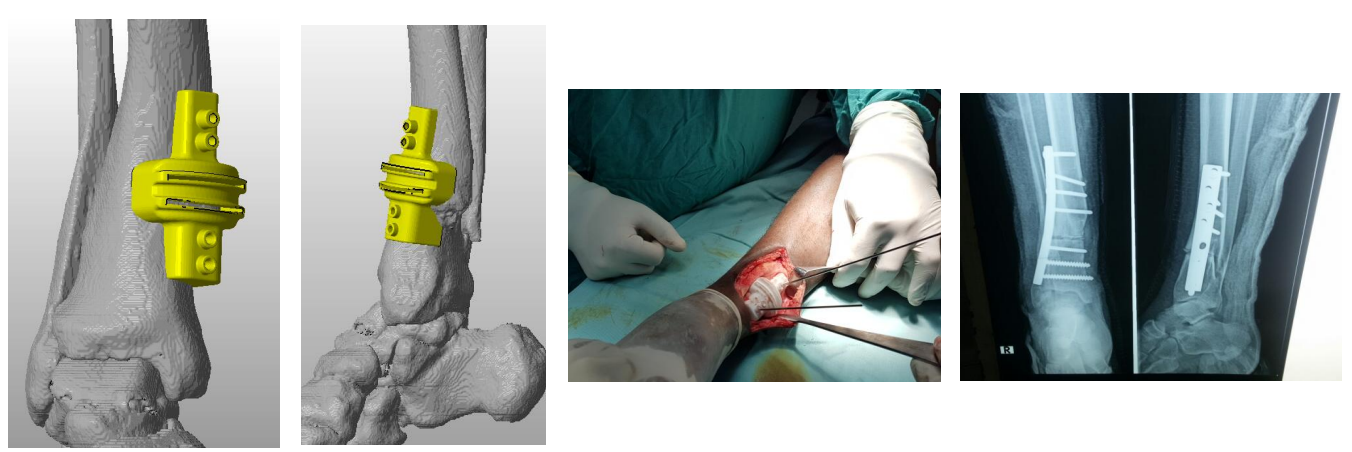

Fig (5): Surgery planning of tibial shaft deformity 